\title{
TU/e EnNHOUN

\section{Experimental set-up for testing alignments and measurement stability of a metrology system in Silicon Carbide for GAIA}

\section{Citation for published version (APA):}

Veggel, van, A. A., Wielders, A. A., Brug, van, H., Rosielle, P. C. J. N., \& Nijmeijer, H. (2005). Experimental setup for testing alignments and measurement stability of a metrology system in Silicon Carbide for GAIA. In A. E. Hatheway (Ed.), Optomechanics 2005, San Diego, California (pp. 1-12). (Proceedings of SPIE; Vol. 5877). SPIE. https://doi.org/10.1117/12.614031

DOI:

10.1117/12.614031

Document status and date:

Published: 01/01/2005

\section{Document Version:}

Accepted manuscript including changes made at the peer-review stage

\section{Please check the document version of this publication:}

- A submitted manuscript is the version of the article upon submission and before peer-review. There can be important differences between the submitted version and the official published version of record. People interested in the research are advised to contact the author for the final version of the publication, or visit the $\mathrm{DOI}$ to the publisher's website.

- The final author version and the galley proof are versions of the publication after peer review.

- The final published version features the final layout of the paper including the volume, issue and page numbers.

Link to publication

\section{General rights}

Copyright and moral rights for the publications made accessible in the public portal are retained by the authors and/or other copyright owners and it is a condition of accessing publications that users recognise and abide by the legal requirements associated with these rights.

- Users may download and print one copy of any publication from the public portal for the purpose of private study or research.

- You may not further distribute the material or use it for any profit-making activity or commercial gain

- You may freely distribute the URL identifying the publication in the public portal.

If the publication is distributed under the terms of Article 25fa of the Dutch Copyright Act, indicated by the "Taverne" license above, please follow below link for the End User Agreement:

www.tue.nl/taverne

Take down policy

If you believe that this document breaches copyright please contact us at:

openaccess@tue.nl

providing details and we will investigate your claim. 


\title{
Experimental set-up for testing alignment and measurement stability of a metrology system in Silicon Carbide for GAIA
}

\author{
Mariëlle van Veggel ${ }^{\mathrm{a} a}$, Arno Wielders ${ }^{\mathrm{b}}$, Hedser van Brug ${ }^{\mathrm{b}}$, Nick Rosielle ${ }^{\mathrm{a}}$, Henk Nijmeijer ${ }^{\mathrm{a}}$ \\ ${ }^{a}$ Technische Universiteit Eindhoven, Department of Mechanical Engineering, P.O. Box 513, \\ 5600 MB Eindhoven, The Netherlands; \\ ${ }^{\mathrm{b}}$ TNO Science and Industry, division Opto-Mechanical Instrumentation, Stieltjesweg 1, 2628 CK \\ Delft, The Netherlands
}

\begin{abstract}
The GAIA satellite will make a 3-D map of our Galaxy with measurement accuracy of 10 microarcseconds using two astrometric telescopes. The angle between the lines-of-sight of the two telescopes will be monitored using the Basic Angle Monitoring system with 1 microarcsecond accuracy. This system will be an interferometer consisting of a number of small mirrors and beam splitters in Silicon Carbide. Silicon Carbide has very high specific stiffness and very good thermal properties (low CTE and high conductivity). It also is a very stable material. A possible concept design for this Basic Angle Monitoring system is subject of a PhD study performed at the Technische Universiteit Eindhoven and TNO Science and Industry (The Netherlands). To prove that this concept design meets the alignment and measurement stability requirements, the GAIA extreme stability optical bench is developed. It will consist of a fourfold Michelson interferometer with four separate optical paths, which will measure the stability of the optical bench and the individual optical components. Also thermal cycling experiments and vibrations tests will be performed. 'Absolute' position measurements of the optical components with respect to the optical bench after the vibrations test will be performed using markers. The GAIA extreme stability optical bench will be placed in a vibration damped vacuum tank in order to imitate the highly stable L2 space environment. The goal is to obtain the first results early 2006.
\end{abstract}

Keywords: GAIA, Silicon Carbide, metrology, stability, measurement

\section{INTRODUCTION}

ESA is currently preparing a satellite called GAIA (Global Astrometric Interferometer for Astrophysics). GAIA will be capable of measuring the position and velocity of a billion stars in 6 years with a positional accuracy of 10 microarcseconds ( $\mu$ as) and a velocity accuracy of $1 \mathrm{~km} / \mathrm{s}$ at magnitudes as low as $15^{1}$. The preliminary design of the GAIA satellite (of which an artistic impression is shown in Fig. 1) consists of three modules: the sun shield, the service module (SVM) and the payload module (PLM). The payload module accommodates the scientific instrumentation (Fig. 2):

- Two identical astrometric telescopes, which measure the position and velocity of stars. The basic angle (the angle between the lines of sight of the telescopes) will be $\sim 100^{\circ}$.

- Focal plane assembly: a large CCD camera, which collects the light from the telescopes.

- Spectrometric instrument, which determines the radial velocity and the chemical composition of stars.

To ensure mechanical and thermal stability in the PLM, it has been decoupled from the SVM as much as possible by placing the optical bench on six struts in three pairs. The maximum diameter of the optical bench is $3 \mathrm{~m}$. The payload module will be covered with a multi-layer insulation tent providing insulation from external influences.

The temperature of the PLM will be $140 \mathrm{~K} \pm 0.1 \mathrm{mK}$. The main heat source in the PLM will be the focal plane. Vibrations only occur during periodic engine bursts. The satellite will rotate around its axis every 6 hours, which means the telescopes make a scanning movement with respect to the stars.

*a.a.v.veggel@tue.nl; phone ++31 (0)40247 4925; fax ++31 (0)40 246 1418; www.tue.nl 


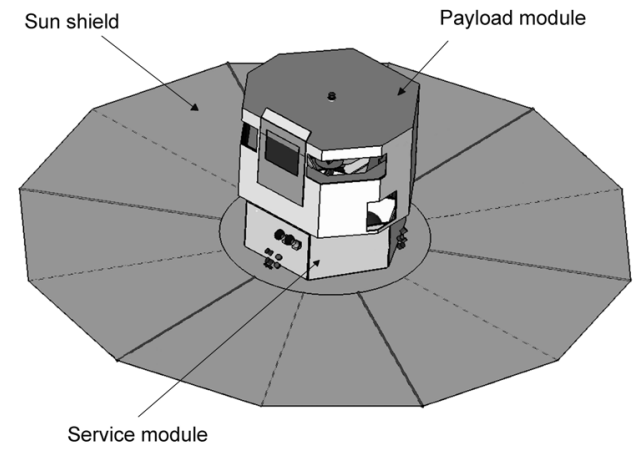

Fig. 1 Impression of the GAIA satellite ${ }^{2}$

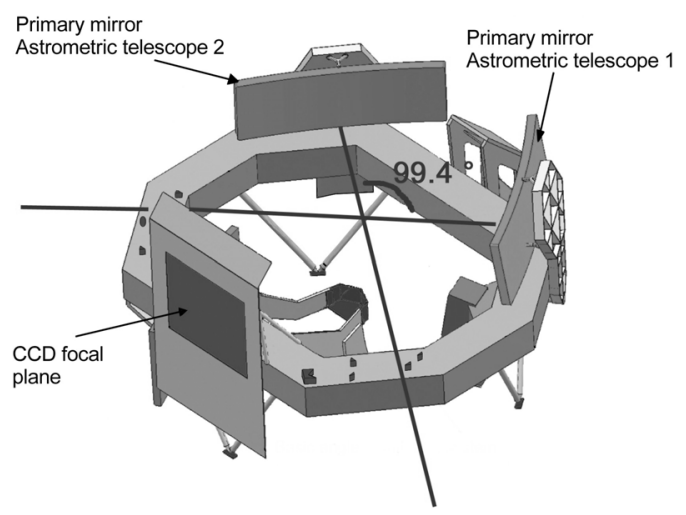

Fig. 2 Impression of the payload module of GAIA with scientific instruments ${ }^{2}$

\subsection{Choice of material}

During operation, the scientific instruments not only need to stay aligned for 6 years, the scientific instruments also need to have a high stability level in the cycle time of 6 hours in order to reach the $10 \mu$ as accuracy of measured positions of stars. For this reason the PLM will be made almost completely of Silicon Carbide ( $\mathrm{SiC})$, because it is a lightweight material $\left(\rho=3100 \mathrm{~kg} / \mathrm{m}^{3}\right)$, it is very stiff $(E=420 \mathrm{GPa})$, it has low thermal expansion $\left(\alpha=2 \cdot 10^{-6} / \mathrm{K}\right)$ and it has high thermal conductivity $(\lambda=170 \mathrm{~W} / \mathrm{m} / \mathrm{K})$. It is also a chemically stable material.

\subsection{Basic Angle Monitoring system}

To be able to link the measured positions of stars of one telescope, to the measured positions of stars of the other telescope, it is very important to know the angle between both telescopes. This "basic angle" is measured prior to launch. Maximum variations of 15 prad are expected on the basic angle in the 6-hour cycle, but they may be larger. Therefore, the satellite will most likely be equipped with a metrology system, which will specifically measure the variations of the basic angle with an accuracy of 5 prad, with the goal of mathematically correcting for these variations in the measured positions of stars. The full name of this metrology system is the Basic Angle Monitoring (BAM) system. The BAM system will also be made of $\mathrm{SiC}$.

Fig. 3 shows the top view of the measurement principle of the BAM system in GAIA. The octagonal structure, on which the scientific instruments are mounted, is visible. The primary mirrors of both telescopes are shown on the left and the right. Bars 1 and 2, which are facing the primary mirrors of the telescope on the opposite side of the octagonal structure, contain the BAM system. Essentially on bar 1, a laser source and collimator send out a laser beam, which is divided into two coherent laser beams on bar 1 (beams 1 and 2 in Fig. 3), which are send to astrometric telescope 1. The laser beam is also split into a pair of coherent laser beams, which travel to bar 2. The beams arriving at BAM bar 2 will be redirected using mirrors toward astrometric telescope 2 (beams 3 and 4 in Fig. 3). Each beam pair will finally interfere like double slit refraction, creating two interference patterns on the focal plane assembly (Fig. 4). If one of the fringe patterns shifts with respect to the other fringe pattern, this is a measure for the change in angle between the telescopes. To be able to reach the $10 \mu$ as accuracy in star position measurements, it is necessary to measure the angle variation between both telescopes with an accuracy of $1 \mu$ as. A small rotation of the primary mirror of the telescope causes the fringe pattern to shift. Any rigid body movement of the bar 1 or bar 2 should have no influence on the differential fringe pattern shift.

\subsection{Requirements}

The alignment accuracy, alignment stability and measurement stability requirements of the individual mirrors and beam splitters of the BAM system are shown in Table 1. The alignment accuracy requirements are based on an optical path length requirement, which ensures centering of the white fringe in the interference pattern. The angle requirement is 
based on a required $90 \%$ overlap of the interfering beams. The stability requirement of 1.1 pm during a 6 hours measurement cycle is a combined requirement with a laser wavelength stability requirement of $10^{-9} \delta \lambda / \lambda$.

A design for the basic angle monitoring system has been made, and stability of the optical components should be proven experimentally. In this paper the development of the experiments with an extreme stability optical bench will be discussed. In section 2 the goals and measurement plan of the experiments will be discussed. Section 3 will deal with the optical design of the set-up. In section 4, the mechanical design concepts will then be presented. Finally, the paper will end with a discussion on future research.

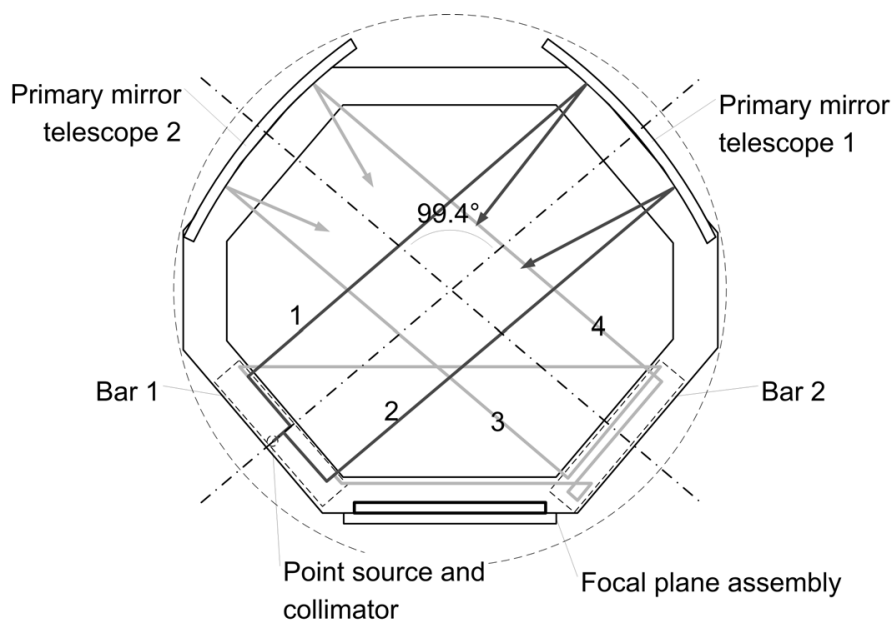

Fig. 3 Measurement principle of the Basic Angle Monitoring system

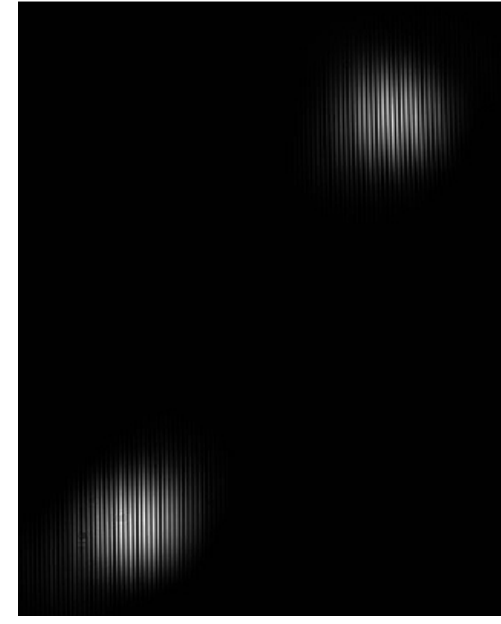

Fig. 4 The interference patterns of two pairs of interfering laser beams

Table 1 Alignment and stability requirements of individual optical components of the BAM system

\begin{tabular}{|l|l|l|l|}
\hline $\begin{array}{l}\text { Parameter for one } \\
\text { component }\end{array}$ & $\begin{array}{l}\text { Alignment accuracy of fine } \\
\text { aligned component }\end{array}$ & $\begin{array}{l}\text { Alignment stability } \\
\text { requirement over 6 years }\end{array}$ & $\begin{array}{l}\text { Stability requirement over 6 } \\
\text { hours }\end{array}$ \\
\hline Position & $5 \mu \mathrm{m}$ & $1 \mu \mathrm{m}$ & $1.1 \mathrm{pm}$ \\
\hline Angle & $6 \mu \mathrm{rad}$ & $1.2 \mu \mathrm{rad}$ & $0.16 \mu \mathrm{rad}$ \\
\hline
\end{tabular}

\section{GOALS AND MEASUREMENT PLAN}

\subsection{Goals}

As explained in the introduction, the important issues for the BAM system are the alignment stability and measurement stability. These requirements are each equally important and influenced by different events in the lifetime of the satellite. The alignment stability should be maintained during the entire measurement lifetime of the satellite from the moment the alignment is achieved. The most extreme events, that could negatively influence alignment stability of the BAM system, are the launch vibrations and shock and the cooling of the BAM system from room temperature to $140 \mathrm{~K}$.

The measurement stability requirements are requirements that should be met during each 6 hour measurement cycle, in order to reach the required measurement accuracy. The measurement stability can be influenced by thermal variations of the bench and micro-vibrations induced by the thrusters, etc.

The most sensitive locations within the mechanical design to alignment and measurement instability, are considered the bonding location of the independent Silicon Carbide bodies. In the mechanical design of the BAM system connection of multiple $\mathrm{SiC}$ bodies is made between the opto-mechanical components and the optical bench. This means that the most important measurements should be focused on the stability of an individual mirror and beam splitter with respect to the optical bench. 
Secondly the measurement of the stability of the optical bench itself is important, because the light will need to travel a large distance across the optical bench. These considerations lead to the goals of the GAIA extreme stability optical bench, which are to measure:

- $\quad$ alignment stability of the individual optical components by measuring the 'absolute' position of the optical components with respect to the optical bench before and after vibrations testing and also by measuring the 'absolute' position of the optical components after thermal cycling from $293 \mathrm{~K}$ to $140 \mathrm{~K}$. The accuracy of these measurements should be $1 \mu \mathrm{m}$ in position and $1 \mu \mathrm{rad}$ in angle based on the alignment stability requirement of the BAM system (Table 1)

- $\quad$ measurement stability of the individual optical components by measuring changes in positions and angle of two optical components at $140 \mathrm{~K}$ in vacuum in a chamber damped against mechanical vibrations and kept thermally stable to $0.1 \mathrm{mK}$. The accuracy of these measurements should be $1 \mathrm{pm}$ in position and $0.1 \mu \mathrm{rad}$ in angle, based on the measurement stability requirements of the BAM system (Table 1).

\subsection{Measurement plan}

The measurements will be performed in the following sequence. First the optical components will be aligned on the optical bench in ambient temperature and pressure. If this has been achieved, the 'absolute' position of the optical components with respect to the optical bench is measured. These measurements are used as a reference.

In the next step the GAIA extreme stability optical bench is placed in the vibration damped vacuum chamber ${ }^{4}$. The optical bench is equipped with temperature sensors at different places on the optical bench to be able to measure temperature gradients across the optical bench with $0.01 \mathrm{mK}$. The temperature sensors should be able to function at 140 $\mathrm{K}$. The laser is initialized and the fringe detector will measure the relative OPD movement of the mirrors. The running system is left to stabilize. Then the stability of the optical components during long period (a few hours) measurements will be measured at ambient pressure and temperature. This measurement also involves pressure, temperature and vibration measurements in order to have a view on the environmental stability. If the measurement results are within the expected range of the order $0.1 \mathrm{~nm}$, the pressure will be reduced to 0.1 bar vacuum and the stability measurements will be repeated. The goal is to achieve a highly stable environment, which resembles as well as possible the environmental conditions in L2. The most important aspect here is the temperature stability of $0.1 \mathrm{mK}$. In this case the OPD stability of the optical paths should be below $50 \mathrm{pm}$.

The running set-up is cooled down to $140 \mathrm{~K}$ in the fourth step. The temperature inside the chamber is kept at $0.1 \mathrm{mK}$. By counting the number of fringes that pass by the detector, the displacement of the optical components with respect to the 'absolute' positions measured after the vibrations test can be determined. This displacement should stay below $1 \mu \mathrm{m}$ and the rotation should stay below $1 \mu \mathrm{rad}$.

The running system is left to stabilize for the final stability measurement. The stability of the optical components during long periods (a few hours) will be measured. In this case the OPD stability of the optical paths should be below 12.5 pm and the relative stability of the BAM optical components should be below $1 \mathrm{pm}$. The angular stability should remain below $0.1 \mu \mathrm{rad}$.

In the final step the optical bench with aligned optical components will be subjected to random vibrations at a vibrating table.

After this experiment, the 'absolute' position of the optical components with respect to the optical bench is measured. These measurement results will be compared to the 'absolute' position measurements that were made before the vibrations test. If the alignment is maintained with $1 \mu \mathrm{m}$ and $1 \mu \mathrm{rad}$ accuracy, the measurements can proceed to the next step. Otherwise, steps should be taken to make sure that alignment is maintained, and steps 1 to 5 should be executed again.

\section{OPTICAL DESIGN AND REQUIREMENTS}

The alignment stability measurements for the vibrations tests will be performed using markers on both the optical bench and the mirror and beam splitter. The alignment stability measurements during thermal cycling tests and the measurement stability experiments will be relative interferometric measurements using a Michelson interferometer. First 
the Michelson interferometer for the measurement stability experiments will be discussed in the next section. Then the alignment stability measurements, using markers will be discussed.

\subsection{Measurement stability experiments}

\section{Optical bench layout for OPD measurements}

The goal of the measurement stability experiments is to measure the stability of an individual mirror with respect to the optical bench. The set-up consists of four Michelson interferometers, combined in 3 mirrors and one beam splitter. The light source is a He-Ne laser (wavelength $\sim 632.8 \mathrm{~nm}$ ).

The optical design of the setup is depicted in Fig. 5. Mirrors MM1 and MM2 represent the 'monolithic' mirrors, which are fixed to the optical bench such that they are considered infinitely stable with respect to the optical bench. Mirror BM is the BAM mirror, S represents the BAM beam splitter. These are both adjustable and will be used in the proposed BAM system of GAIA. Mirrors SM1 and SM2 are deposited mirrors on beam splitter S.
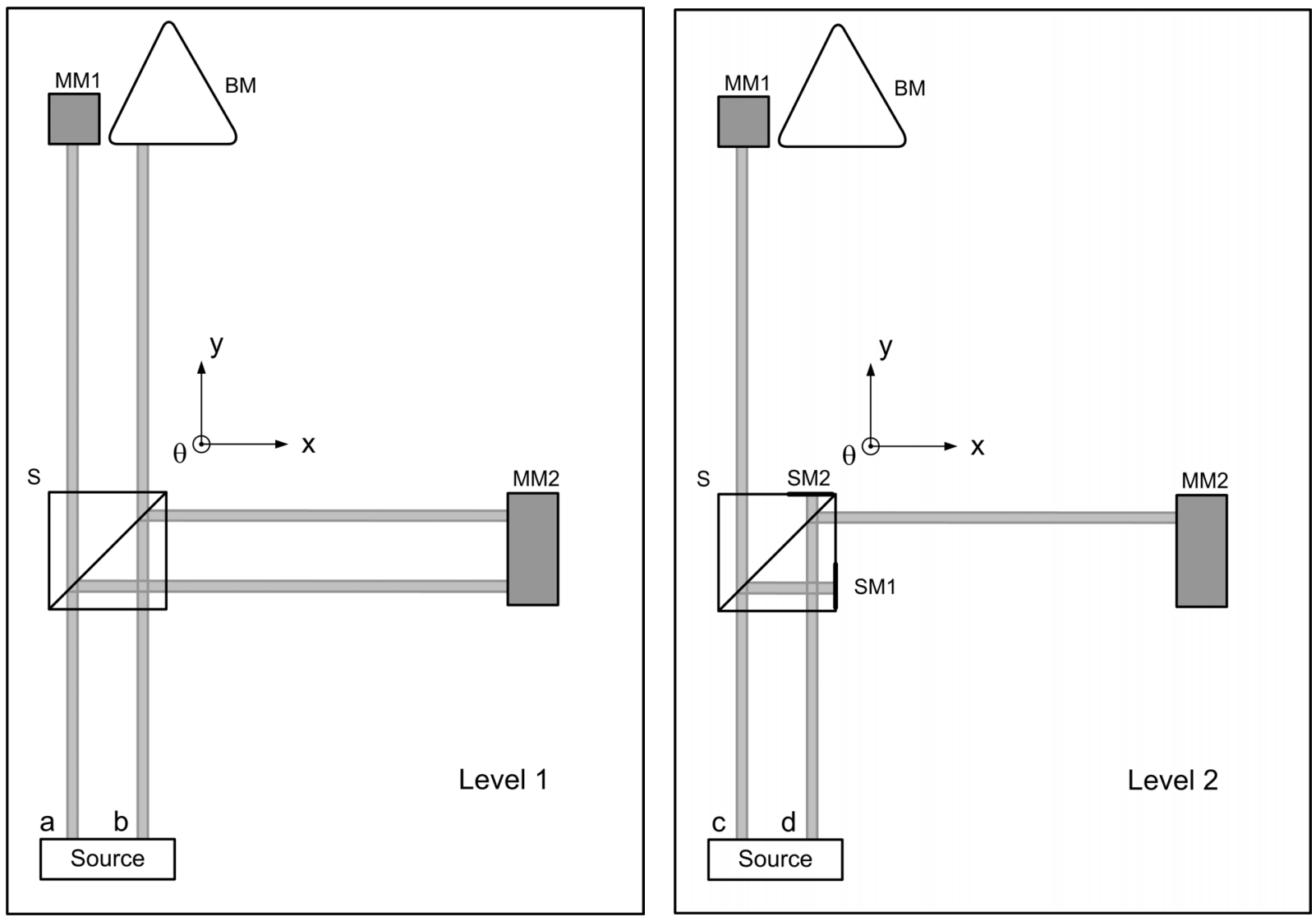

Fig. 5 Optical layout of the optical bench

In Fig. 5 it is indicated that four laser beams will enter the interferometer core. Two of these beams enter at level 1 , the lower level. The other two beams enter at level 2, the upper level. The arrangement of the beams coming from the source is shown in Fig. 6 a). The four beams are coherent and each have a diameter $\mathrm{D}=3 \mathrm{~mm}$.

At level 1, laser beam ' $a$ ' measures the optical path length change between MM1-S and MM2-S. The two arms have nearly the same length, making them each virtually insensitive to wavelength instabilities of the laser. The optical path length of beam ' $a$ ' is influenced by the instability in the homogeneous expansion of the optical bench, the positional and angular instability of the beam splitter S and the shape instability of beam splitter S. Laser beam ' $b$ ' measures the optical path length change between BM-S and MM2-S. The optical path length of beam ' $b$ ' is influenced by the same effects as 
beam 'a', but there is also an additional effect, which is the positional instability of the BAM mirror BM. Subtracting the data obtained via beams ' $a$ ' and ' $b$ ' gives a value on the instability of the BAM mirror with respect to mirror MM1.

At level 2, laser beam 'c' measures the optical path length change between MM1-S and SM1-S. The arms of this beam are not equal, making this measurement sensitive to wavelength instabilities. The optical path length change measured by beam 'c' is thus influenced by wavelength instabilities of the laser, by the expansion of the optical bench, the positional and angular instability of the beam splitter $S$ and the shape instability of beam splitter S. Laser beam ' $d$ ' measures the optical path length change between MM2-S and SM2-S, which is influenced by the same instabilities as beam 'c'. Subtracting the results obtained via beams ' $c$ ' and ' $d$ ' provides the optical path length change due to inhomogeneous expansion of the optical bench and inhomogeneous expansion of the beam splitter. Note that subtracting the data from beams ' $c$ ' and ' $d$ ' eliminates the sensitivity to laser wavelength instability. If finally, the data from beam 'a' is subtracted from the subtracted data from beams ' $c$ ' and ' $d$ ', the positional instability of the beam splitter remains.

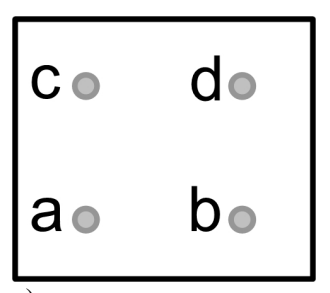

a)

Fig. 6 Beam arrangements in source for a) positional and b) angular stabil

beamsplitter

\section{Measurement of angular measurement stability}

In order to obtain information on the angular stability (tip/tilt) of the mirror surfaces, separate measurements will be conducted in which beam ' $b$ ' will be replaced with three parallel laser beams, each measuring an optical path length change. The beams are arranged in an L-shape, like shown in Fig. 6 b). By comparing the top most position with the bottom position, and the outer left with the outer right, and knowing the separation between these points, the angular variation can be calculated.

\section{Requirements}

The laser used for this Michelson interferometry system is a He-Ne laser, which has a wavelength stability over several hours of:

$$
\frac{\delta \lambda}{\lambda}=2 \cdot 10^{-9}
$$

This wavelength stability dictates for a large part the alignment requirements of the optical components in the set-up.

\section{OPD alignment}

The OPD alignment requirement of the mirrors and the beam splitter is directly related to the wavelength stability of the He-Ne laser. If the assumption is made that the wavelength instability may not cause an apparent optical path change of more than $0.1 \mathrm{pm}$, the OPD alignment of the mirrors and beam splitter with respect to each other, should be:

$$
\Delta L_{\max }=\frac{\delta O P D_{\max }}{\delta \lambda / \lambda}=\frac{0.1 \cdot 10^{-12}}{2 \cdot 10^{-9}} \cong 0.05 \mathrm{~mm}
$$

\section{Source alignment stability}

Apart from laser wavelength changes, also the beam pointing stability is of influence to the results. A pointing error of $\delta \theta$ leads over a path length difference $\Delta \mathrm{L}$ and an alignment error $\delta \psi$ between the interferometer arms to an apparent OPD change of: 


$$
\delta O P D=\left|\frac{2 \cdot L_{2}}{\cos (\delta \theta)}-\frac{L_{1}}{1-\tan (\delta \theta) \cdot \tan (\delta \psi)} \cdot\left\{\sqrt{\tan ^{2}(\delta \theta)+1}+\frac{1}{\cos (\delta \theta+2 \cdot \delta \psi)}\right\}\right|-\left|2 \cdot L_{2}-L_{1} \cdot\left(1+\frac{1}{\cos (2 \cdot \delta \psi)}\right)\right|
$$

The plots in Fig. 7 show independently the OPD change, with increasing pointing error for a pre-existing OPD and for a pre-existing angular alignment error of one mirror with respect to the beam splitter and the other mirror. The OPD change with the same pointing instability is a factor $10^{4}$ smaller, than for the angular alignment error. The angular alignment of the mirrors is thus linked with the source alignment stability. In the most realistic case the angular alignment error and the source alignment stability are both $1 \mu \mathrm{rad}$ for a maximum error of $5 \mathrm{pm}$.

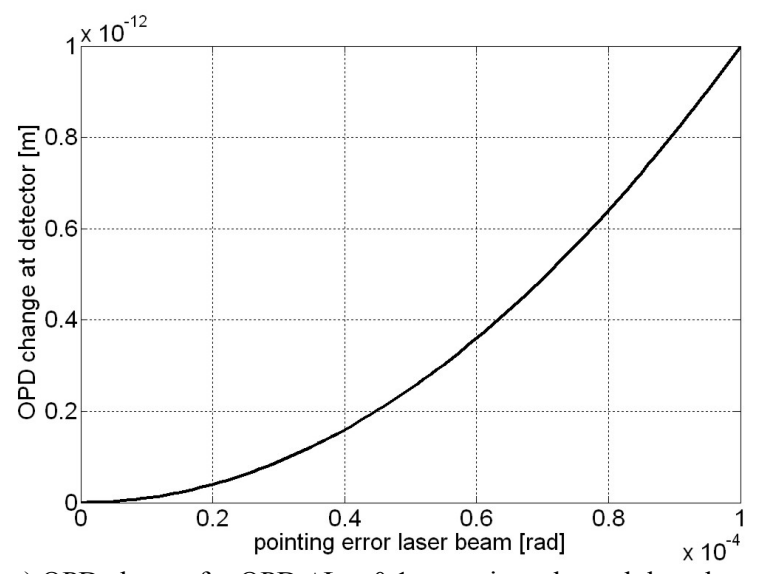

a) OPD change for OPD $\Delta \mathrm{L}=0.1 \mathrm{~mm}$, since the path length $\mathrm{L}_{10}=250 \mathrm{~mm}$ and $\mathrm{L}_{20}=250.1 \mathrm{~mm}, \delta \psi=0 \mathrm{rad}$.

Fig. 7 OPD changes for increasing pointing error $\delta \theta$.

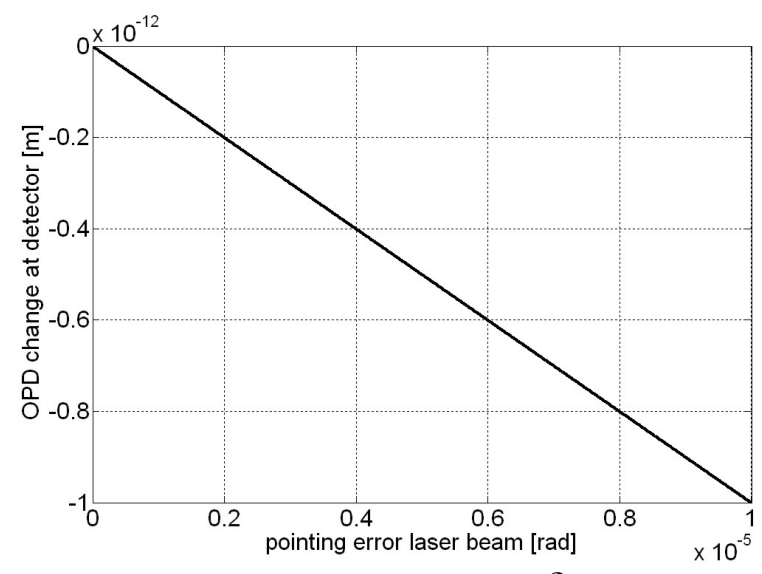

b) OPD change for angular alignment error $\delta \psi=1 \mu \mathrm{rad}$, and path lengths $\mathrm{L}_{10}=\mathrm{L}_{20}=250 \mathrm{~mm}$

\section{Angular alignment}

The fact that the angular alignment error may be maximally $1 \mu \mathrm{rad}$, means that one of the mirrors or the beam splitter for each beam should be aligned with this accuracy in the final alignment stage. This does not mean that all the mirrors need to be aligned with this accuracy, since accurate alignment of just one mirror can correct for errors created by the other optical components. The goal is to align them with $1 \mathrm{mrad}$ accuracy.

\section{Intensity stability of the laser}

The detector uses heterodyne detection to measure any path length change, which means the measurement is performed by a combination of counting the fringe minima, and measuring the phase between the interfering beams. This means that the system is nearly insensitive to intensity variations of the laser source.

\section{Phase resolution of the detector}

If the path length difference between the beams is changed one wavelength $(\lambda=632.8 \mathrm{~nm})$, one dark fringe has passed. A shift of one mirror of $1 \mathrm{pm}$, will cause an OPD change of $2 \mathrm{pm}$, which is thus $2 \cdot 10^{-3} / 632.8=1 / 316400$ fringe. The phase detector should therefore have a resolution of $2 \pi / 316400 \mathrm{rad}=2 \cdot 10^{-5} \mathrm{rad}$.

\section{Detector alignment stability}

The detector alignment stability during measurements should be $0.1 \mathrm{mrad}$, such that the detector can keep track of the signal.

\section{Mirror and beam splitter flatness}

The allowed angle between two beams is about $0.1 \mathrm{mrad}$ (dictated by the detector unit of the interferometer) which directly gives a flatness requirement on all mirrors and beam splitters. For the mirror surfaces in the system this means that $\lambda / 10$ suffices and for the transmitted surfaces $\lambda / 4$ should be enough. 


\section{Sampling frequency}

The detector and software will be capable of measuring the phase with a sampling rate of $60 \mathrm{MHz}$. Since the proposed GAIA BAM system will not register any variations of the basic angle with a frequency higher than approximately $1 \mathrm{~Hz}$, a batch of $60 \cdot 10^{6}$ samples will be used to average to picometer detector resolution. The theoretical resolution, taking into account the error budgets of the stability optical bench is $5 \mathrm{pm}$.

\section{Resolution of angular stability measurements}

The distance between the three beams for the angular stability measurements will be $10 \mathrm{~mm}$. Since the distance changes will be measured with a resolution of about $2 \mathrm{pm}$, the angular resolution will be below $1 \mathrm{nrad}$, which is far better than is required. The error created by a deviation of the $10 \mathrm{~mm}$ distance between the beams will be less than $0.001 \mathrm{pm}$, which is negligible.

\subsection{Alignment stability measurements}

The alignment stability measurements are performed using differential measurements. In which the distance of a reference plane on the optical bench to the BAM mirror is measured before and after mechanical vibrations. The differential measurements will be performed using markers which are present on the optical bench and the mirror.

\section{MECHANICAL DESIGN}

The mechanical design of the GAIA extreme stability optical bench consists of an environmental chamber, and the design of the GAIA extreme stability optical bench itself. Both are discussed in this section.

Top view

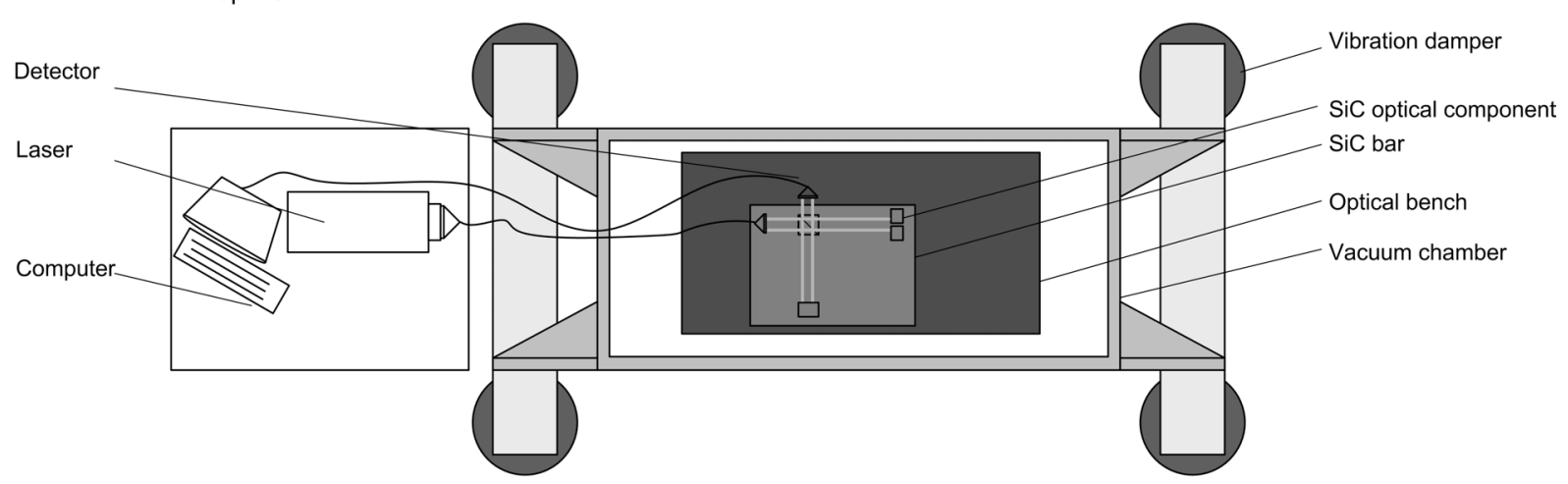

Side view

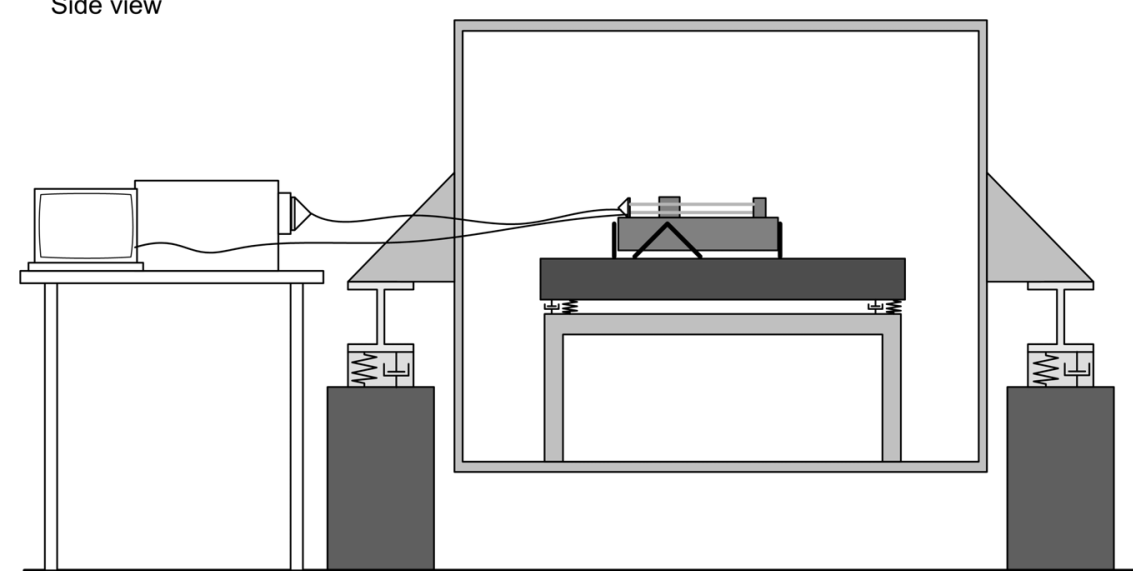

Fig. 8 GAIA extreme stability optical bench in environmental chamber 


\subsection{Environmental chamber}

The environmental chamber will be used to approach as well as possible the conditions in the L2 point. Conditions in the L2 will be very stable in high vacuum, at $140 \mathrm{~K} \pm 0.1 \mathrm{mK}$ and only microvibrations $(<1 \mu \mathrm{N} / \sqrt{ } \mathrm{Hz})$ will exist. The environmental chamber will therefore be a vibration damped, cryogenic vacuum chamber. The environmental chamber will be in a conditioned room to achieve optimal stability of the laser.

A schematic view of the environmental chamber is shown in Fig. 8. The environmental chamber consists of a chamber, which is placed on four seismic vibration dampers. The vibration damped bench is mounted on acoustic dampers in the vacuum chamber to damp any acoustic vibration which resonate the walls of the vacuum tank.

The laser will be outside the vacuum chamber. The laser beam is polarized in two orthogonal directions. The light will be send into the vacuum chamber using optical fibers via a pressure flange, which are polarization-conserving for both orthogonal polarization directions.

The GAIA extreme stability optical bench will be mounted on the vibration damped bench of the vacuum chamber. Since the alignment of the detector is not critical, the detector will not be on the GAIA extreme stability optical bench. The detector will be positioned on the aluminium vibration damped optical bench of the vacuum tank. The advantage of this is that the heat produced by the detector can be isolated in conduction from the GAIA extreme stability optical bench, in order to increase the temperature stability of the measurements. The information obtained by the detector is send via wires to the computer outside the vacuum chamber.

\subsection{GAIA extreme stability optical bench}

An impression of the actual GAIA extreme stability optical bench is shown in Fig. 9. It shows the optical configuration shown in Fig. 5 in a 3D view. It will be constructed in Silicon Carbide for a large part, like will be the case for the actual GAIA payload module and the BAM system.

\section{Optical bench}

The optical bench on which the optical components will be mounted (Fig. 9), is dimensioned 500 by $400 \mathrm{~mm}$ for the top area and $100 \mathrm{~mm}$ for the height. This is based on a realistic optical path length between two optical components for the BAM system. The mass of the optical bench is circa $10 \mathrm{~kg}$, which is based on the mass-volume ratio of the optical bench of the GAIA satellite. The optical bench will be a ventilated closed back honeycomb structure, where the closed box structure provides for bending stiffness of the optical bench and the honeycomb structure provides for the flatness stability of the optical bench mounting surface.

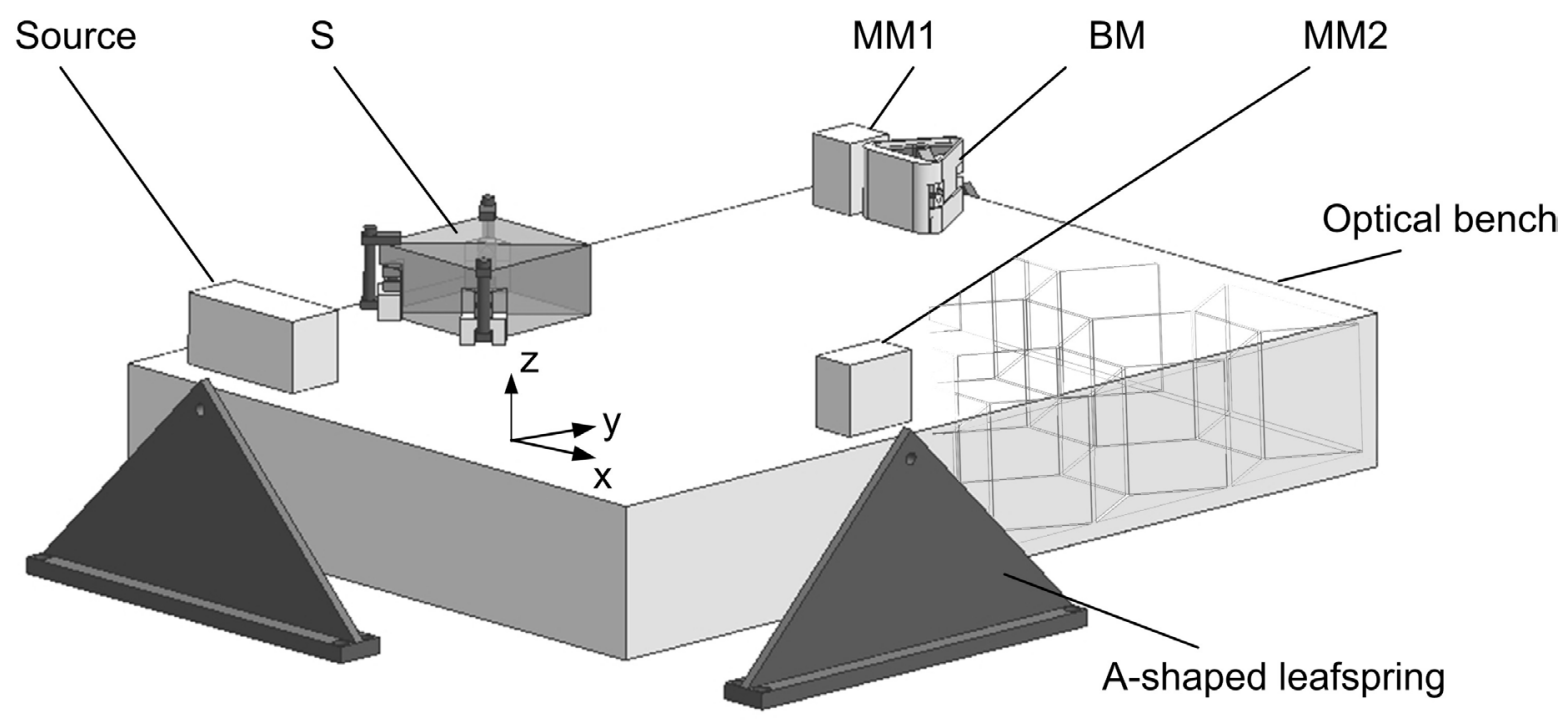

Fig. 9 Impression of the GAIA extreme stability optical bench 
The honeycomb structure and wall thicknesses are optimized by performing finite element calculations with a modal loadcase and a $20 \mathrm{G}$ load in three directions simultaneously loadcase. The lowest eigenfrequency is maximized and the misalignment of the mirrors is minimized.

The $\mathrm{SiC}$ optical bench is mounted onto the aluminium vibration damped bench of the vacuum chamber using A-shaped leafsprings, which can accommodate for the thermal expansion difference between the SiC optical bench and the aluminium, which will be as much as $1.7 \mathrm{~mm}$ over the entire length of the SiC optical bench.

\section{Source holder}

The source holder will hold the optical fibers and lenses, which send the light in $3 \mathrm{~mm}$ diameter laser beams to the interferometer. The source holder will be on the GAIA extreme stability optical bench. To make it insensitive to pointing instabilities, the pinhole principle will be used.

\section{'Monolithic' mirrors}

The 'monolithic' mirrors MM1 and MM2 should preferably be monolithically made with the optical bench of the system. Although this would be considered the most stable solution, the manufacturability of the mirrors with $\lambda / 10$ flatness at right angle with the surface of the optical bench is low. It is therefore necessary to mount the mirrors on the optical bench using brazing or Hydroxide Catalysis Bonding and consider this bonding method infinitely stable.

\section{BAM mirror}

The design of the BAM mirror is shown in Fig. 10. It should be aligned with an accuracy of $1 \mu$ rad in angle and $1 \mu \mathrm{m}$ in position both on the BAM system. This is hardly possible to achieve using bonding methods like brazing or Hydroxide Catalysis Bonding. Therefore a tensioning option has been chosen. The choice of design has been based on two arguments:

- The tensioning interface should be used for alignment purposes

- The tensioning interface should be chosen such that as any tensioning force variations, cause as little as possible misalignment.

The design (Fig. 10) has a thermal centre due to the fact that the mirror is suspended on three V-grooves-ball combinations, which are oriented towards the mirror surface. Alignment is performed by fixing the V-grooves-ball combinations during alignment but shifting the mirror over the flat surface of the upper V-grooves. A central spider is tensioned after achieving alignment.

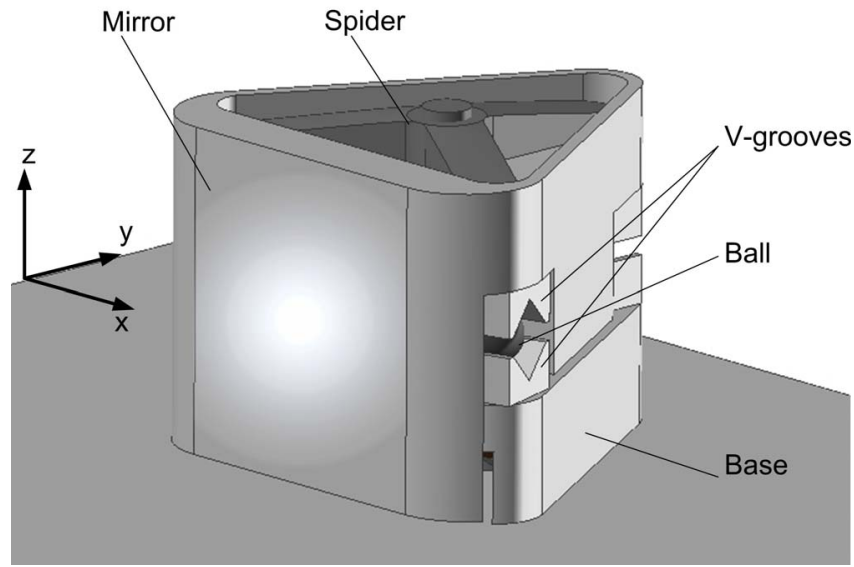

a) $3 \mathrm{D}$ view

Fig. 10 Impression of the BAM mirror
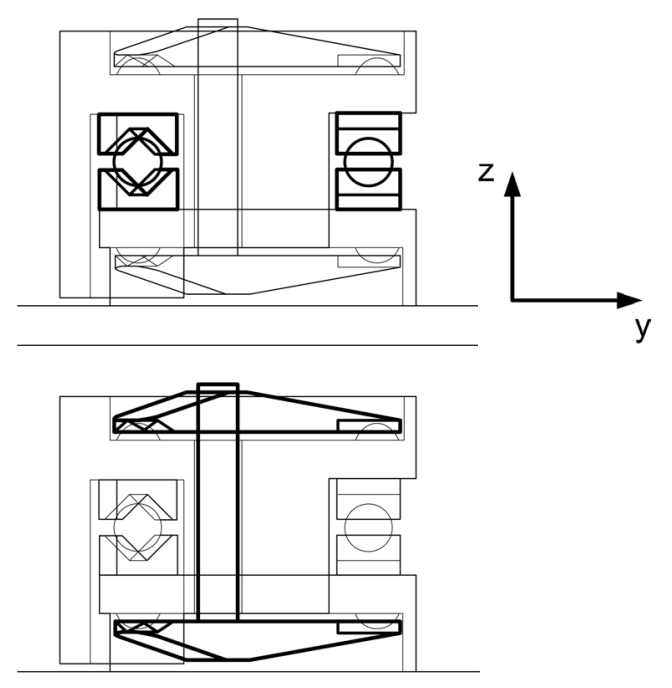

b) 2D drawings with highlighted V-groove ball combinations (upper) and spider (lower) 


\section{BAM beam splitter}

The beam splitter itself is a polarizing beam splitter (Fig. 11). It is mounted in the same manner as the mirror, on three Vgrooves-ball combinations, arranged in such a manner that the beam splitter is least sensitive to force variation of the tensioning mechanism. The $\mathrm{V}$-grooves are pointed towards the centre of the beam splitter $50 \%$ reflective surface, which functions as the thermal centre of beam splitter mount. Since the light travels through the beam splitter a spider tensioning mechanism is not an option. Three independent tensioning mechanisms, depicted schematically in Fig. 11, will be used. The alignment of the beam splitter is performed in the same way as the mirror.

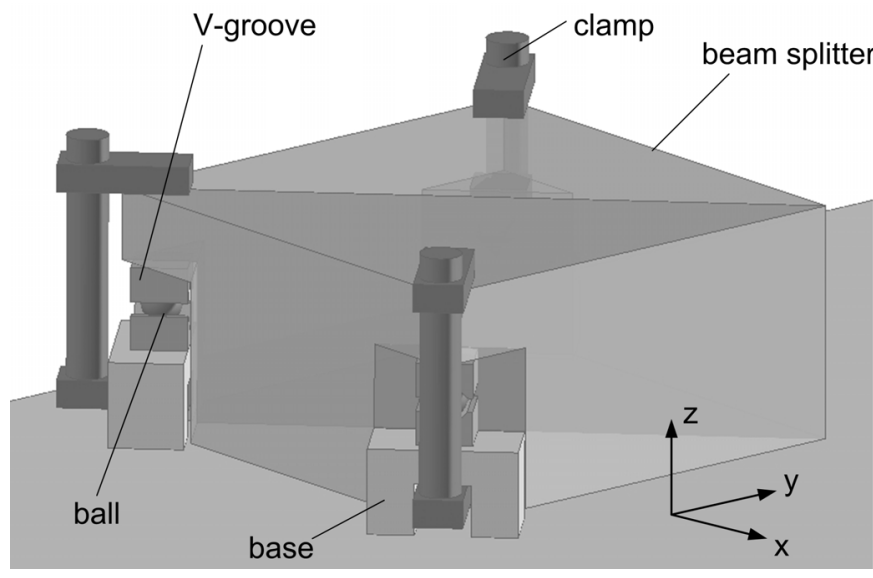

a) $3 \mathrm{D}$ view

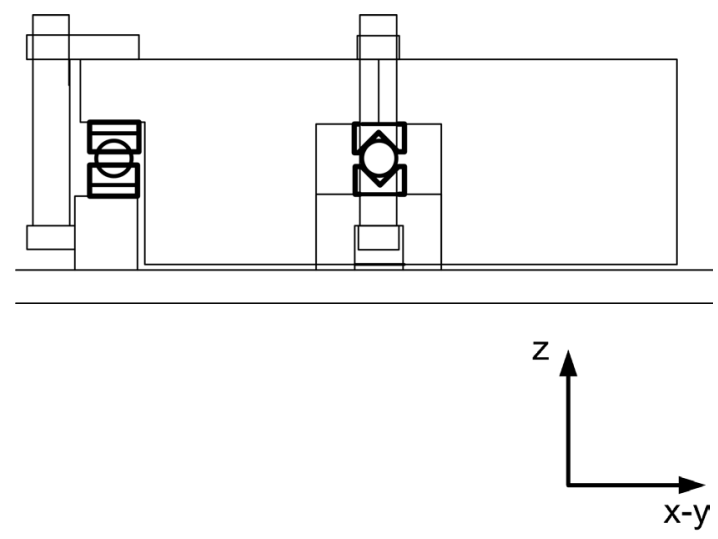

b) 2D drawing with highlighted V-groove-ball combination

Fig. 11 Impression of the BAM beam splitter

\section{Alignment plan}

The alignment of the optical components on the optical bench will be conducted in specific sequence. First the 'monolithic' mirrors will be bonded to the optical bench. The only issue here is to achieve angular alignment with 1 mrad accuracy. This is done by using a metallic template and pushing the mirrors against the template followed by bonding them. In the next phase the beam splitter is placed and aligned. The $1 \mu$ rad angular alignment is achieved using an autocollimator and the $0.1 \mathrm{~mm}$ OPD alignment is achieved by using white light fringes. Next, the BAM mirror is aligned, using white light fringes for the $0.1 \mathrm{~mm}$ OPD alignment and an autocollimator for $1 \mu \mathrm{rad}$ angular alignment with respect to mirror MM1. Finally, the alignment of the source holder will be performed with an accuracy of $1 \mathrm{mrad}$.

\section{CONCLUSIONS}

For GAIA a basic angle monitoring (BAM) system is developed for measuring angular variations of two telescope mirrors. The system will be constructed of Silicon Carbide. The main requirement of the BAM system is positional stability of individual optical components of $1 \mathrm{pm}$. In order to prove that the design meets the requirements measurements will be performed, of which the measurement plan was presented. The measurement plan includes optical stability measurements at room temperature and at $\sim 150 \mathrm{~K}$. The design consists of a fourfold Michelson interferometer with wavelength-split polarized beams. The interferometer is placed in a vibration damped, cooled vacuum tank. It can measure optical bench inhomogeneous expansion, BAM mirror positional and angular instability and BAM beam splitter instability with a theoretical $5 \mathrm{pm}$ accuracy using averaging of 60 million measurement samples per second. Two mirrors are fixed using brazing or Hydroxide Catalysis Bonding and are assumed infinitely stable. They cannot be aligned very accurately. The BAM mirror and beam splitter can be aligned to the required accuracy and are each tensioned mechanically onto the optical bench. The thermal center is created by an interface with three balls in V-grooves, ensuring thermal stability of the optical components.

The design of the GAIA extreme stability optical bench is currently in the phase of final specifications. The goal is to start producing parts by October 2005 and start initial measurements early 2006. 


\section{ACKNOWLEDGEMENTS}

This research is partly supported by the NIVR (Dutch Agency for Aerospace Programs).

\section{REFERENCES}

1. Perryman, M. GAIA in 2003. ESA ESTEC, WG-STATUS-013, Noordwijk, 2004

2. http://sci.esa.int/science-e/www/area/index.cfm?fareaid $=26$ (2005)

3. GAIA SLTRS project team. GAIA system level technical reassessment study final report. Astrium, France, 2002.

4. Snijders, B., Kappelhof, P., Braam, B.C. et al. GAIA testbench: monitoring the basic angle with micro-arcsecond accuracy. SPIE proceedings, vol. 4006, pp. 915-926, 2000 